\title{
GRAPPA 2019 Project Report
}

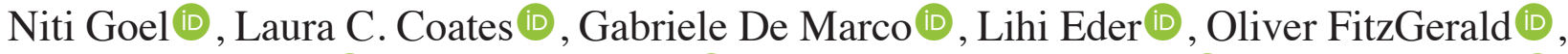 \\ Philip S. Helliwell $\mathbb{D}^{\mathbb{D}}$, Ying Ying Leung $\mathbb{D}^{\mathbb{D}}$, Walter P. Maksymowych ${ }^{\mathbb{D}}$, Philip J. Mease $\mathbb{D}^{\mathbb{D}}$, \\ Mikkel Østergaard (1), Denis O’Sullivan (D), Denis Poddubnyy (D), Christopher T. Ritchlin (1), \\ and Dafna D. Gladman (i)
}

\begin{abstract}
At the 2019 annual meeting of the Group for Research and Assessment of Psoriasis and Psoriatic Arthritis (GRAPPA), members received updates on several ongoing efforts. Among them were updates on research, including the trainee symposium, pilot research grants, and the Collaborative Research Network; GRAPPA's patient research partners; education, including the slide collection; treatment recommendations; and additional work related to advancing the understanding of disease aspects, including the Outcome Measures in Rheumatology (OMERACT)-GRAPPA outcome measure, axial involvement, and ultrasound enthesitis projects; as well as the early psoriatic disease systematic literature review and magnetic resonance imaging. (J Rheumatol Suppl. 2020 June;96:53-7; doi:10.3899/ jrheum.200129)
\end{abstract}

Key Indexing Terms:

PSORIASIS PSORIATIC ARTHRITIS EDUCATION RESEARCH GRAPPA

Members of the Group for Research and Assessment of Psoriasis and Psoriatic Arthritis (GRAPPA) continue to pursue the core objectives of GRAPPA's mission, specifically, to provide education, identify research assessment tools, and pursue research in disease pathophysiology. At the 2019 annual GRAPPA meeting in Paris, France, members received updates on research, including the trainee symposium, pilot research grants, and Collaborative Research Network (CRN); GRAPPA's patient research partners (PRP); education, including the slide collection; treatment recommendations; and additional work related to advancing the understanding of disease aspects, including the Outcome Measures in Rheumatology (OMERACT)-GRAPPA outcome measure, axial involvement, and ultrasound enthesitis projects; as well as the early psoriatic arthritis (PsA) systematic literature review and magnetic resonance imaging (MRI).

\section{Research Committee}

Dr. Oliver FitzGerald reported that the GRAPPA CRN held a very productive premeeting session. The session was held to review progress on the development of an electronic case

From the Duke University School of Medicine, Durham, North Carolina, USA; Department of Orthopaedics, Rheumatology, and Musculoskeletal Sciences, University of Oxford, Oxford; UK National Institute for Health Research (NIHR) Leeds Biomedical Research Centre, Leeds Teaching Hospitals National Health Service (NHS) Trust, Leeds; Leeds Institute of Rheumatic and Musculoskeletal Medicine, University of Leeds, Leeds, UK; University of Toronto and Women's College Hospital, Toronto, Ontario, Canada; Conway Institute for Biomolecular Research, University College Dublin, Dublin, Ireland; Bradford Hospitals UK NHS Foundation Trust, Bradford, UK, Duke-National University of Singapore (NUS) Medical School, Singapore; Department of Rheumatology and Immunology, Singapore General Hospital, Singapore; University of Alberta, Edmonton, Alberta, Canada; Rheumatology Research, Swedish Medical Center and University of Washington School of Medicine, Seattle, Washington, USA; University of Copenhagen and National Hospital (Rigshospitalet), Copenhagen, Denmark; Our Lady's Hospice \& Care Services, Dublin, Ireland; Charité-Universitätsmedizin Berlin, and German Rheumatism Research Centre, Berlin, Germany; Division of Allergy, Immunology, and Rheumatology, University of Rochester Medical Center, Rochester, New York, USA; University of Toronto, Krembil Research Institute, Psoriatic Arthritis Program, University Health Network, Toronto Western Hospital, Toronto, Ontario, Canada.

As part of the supplement series GRAPPA 2019, this report was reviewed internally and approved by the Guest Editors for integrity, accuracy, and consistency with scientific and ethical standards.

N. Goel, MD, Patient Research Partner, Adjunct Assistant Professor, Duke University School of Medicine; L.C. Coates, MB ChB, PhD, Department of Orthopaedics, Rheumatology, and Musculoskeletal Sciences, University of Oxford; G. De Marco, MD, Post-CCT Clinical Research Fellow, UK NIHR Leeds Biomedical Research Centre, Leeds Teaching Hospitals NHS Trust, and Leeds Institute of Rheumatic and Musculoskeletal Medicine, University of Leeds; L. Eder, MD, PhD, Assistant Professor of Medicine, University of Toronto and Women's College Hospital; $O$. FitzGerald, MD, FRCP (UK), FRCPI, Newman Clinical Research Professor, Conway Institute for Biomolecular Research, University College Dublin; P.S. Helliwell, DM, PhD, FRCP, UK NIHR, Institute of Rheumatic and Musculoskeletal Medicine, University of Leeds, and Bradford Hospitals UK NHS Foundation Trust; Y.Y. Leung, MB ChB, MD, Associate Professor, Duke-NUS Medical School, and Department of Rheumatology and Immunology, Singapore General Hospital; W.P. Maksymowych, MB, ChB, FRCPC, Professor of Medicine, University of Alberta; P.J. Mease, MD, Rheumatology Research, Swedish Medical Center and University of Washington School of Medicine; M. Østergaard, $M D, P h D, D M S c$, Professor of Rheumatology at University of Copenhagen and the National Hospital (Rigshospitalet); D. O'Sullivan, BE, Patient Research Partner, Our Lady's Hospice \& Care Services; D. Poddubnyy, MD, MSc (Epi), Professor of Rheumatology, Charité-Universitätsmedizin Berlin, and German Rheumatism Research Centre; C.T. Ritchlin, MD, $\mathrm{MPH}$, Professor of Medicine, Division of Allergy, Immunology, and Rheumatology, University of Rochester Medical Center; D.D. Gladman, MD, FRCPC, Professor of Medicine, University of Toronto, Senior Scientist, Krembil Research Institute, Director, Psoriatic Arthritis Program, University Health Network, Toronto Western Hospital.

Address correspondence to Dr. N. Goel, 4809 Taproot Lane, Durham, North Carolina 27705, USA.

E-mail:agwngw1@gmail.com

Personal non-commercial use only. The Journal of Rheumatology Copyright @ 2020 . All rights reserved. 
report form (eCRF), standardized operating procedures (SOP), and a shared database to be used in CRN studies; to obtain feedback on the SOP needed for sample collection; and to propose an investigator-initiated study that would allow for testing of both the eCRF and SOP. A coordinator for the CRN was appointed and assigned to develop the eCRF and SOP. As a result of the meeting, the CRN committee planned to proceed with a more detailed treatment response study design to guide the development of the eCRF and SOP.

Continued progress on the PsA Biomarkers of Joint DAMage (BioDAM) project was reported, especially with relation to obtaining samples from various industry-sponsored trials in PsA. This included a signed contract with Lilly, with shipping of samples from the ixekizumab PsA program planned in fall 2019; a contract under discussion with Amgen to access samples from the Study of Etanercept And Methotrexate in subjects with Psoriatic Arthritis (SEAM-PsA); and a contract under discussion with Pfizer related to the tofacitinib program to predict treatment response.

GRAPPA is now registered in the European Union (EU; in the Netherlands), allowing GRAPPA to participate in EU-funded research programs. As part of this effort, GRAPPA continues to pursue a psoriatic disease-specific call for research in areas of major unmet need with the EU-based Innovative Medicines Initiative. While the call for proposals was issued in late 2018, it will likely not proceed until 2020. Meanwhile, contact was made with the US National Institutes of Health regarding the possibility of an Accelerated Medicines Partnership-type program in psoriatic disease.

Dr. Christopher T. Ritchlin reported that there were 26 applications for the pilot research grants compared to 22 in 2018. Once again, 3 were awarded at $\$ 25,000$ each to Maria Angioni (Italy) for her study evaluating the pharmacogenetics of treatment response in PsA; Alla Ishchenko (Belgium) on metabolomic profiling in psoriatic disease; and Zhenrui Shi (USA) on the preclinical analysis of CCR6 and CCL20 in mouse and human joints. The Research Committee will send a new request for applications in 2020 with additional information and details provided at that time.

Additionally, results to date from the 3 research projects awarded in 2018 were presented. Dr. Fardina Malik (USA) presented on the effect of medium-chain fatty acid (MCFA) supplementation on PsA and psoriasis. She noted that 39\% of psoriatic disease patients report the use of complementary and alternative medicine. She examined how MCFA supplementation, specifically pure extra virgin coconut oil (PEVCO), regulates psoriatic disease. Theoretically, MCFA in immune regulation could alter gut microbiota, have an antimicrobial effect, modulate $\mathrm{T}$ cell function, increase interleukin (IL)-10 levels, and reduce levels of tumor necrosis factor- $\alpha$ (TNF- $\alpha)$. In the study, patients with active psori- asis with or without PsA began PEVCO supplementation for 6 weeks. They underwent assessment of their skin, joints, and microbiome, and they completed the multidimensional Health Assessment Questionnaire. So far, 14 individuals have been recruited with a median age of 42.5 years $(57 \%$ female). While no changes in clinical disease activity were seen, after 3 weeks of MCFA supplementation, a change in the levels of firmicutes and clostridia was seen in the gut microbiome as well as decreases in the level of IL-22.

Kim Wervers and Dr. Hannah den Braanker (the Netherlands) presented on the response to methotrexate (MTX) and a quest for personalized medicine in PsA. Their objectives were to determine how often a good response to MTX occurs in daily practice in patients with early PsA and what differences exist between responders versus nonresponders. They evaluated 219 PsA patients with 1 year or less of oligo/polyarthritis, of whom 183 (84\%) started taking MTX at the highest tolerated dose within 6 months of diagnosis. Ninety patients (49\%) continued MTX monotherapy at 1 year. Of these patients, $44(49 \%)$ achieved minimal disease activity (MDA) by 6 months, and only 33 maintained MDA status at 1 year. MTX monotherapy was concluded to be insufficient therapy for early PsA. While folate and vitamin B12 levels were similar at baseline, differences between responders and nonresponders included significantly higher baseline levels of IL-23, TNF- $\alpha$, interferon- $\gamma$, granulocyte-macrophage colony stimulating factor, and IL-10. Further, levels of IL-23 and IL-10 evolved differently with MTX monotherapy in responders versus nonresponders. Further work is ongoing to characterize the patients.

Philip S. Helliwell presented Graham Chapman's project on behalf of the EffeCt of Psoriatic Arthritis on Plantar shEar Stress (ESCAPES) study group. This ongoing study aims to examine the hypothesis that abnormal shear stresses in the foot are a contributory factor in the development of dactylitis of the toes in PsA. As part of this project, a new custom-made shear stress device was manufactured and is now ready for laboratory in vivo measurements. The project is planned to be completed by summer 2020 .

\section{MRI}

Dr. Walter P. Maksymowych and Dr. Mikkel Østergaard presented an update on the ongoing work with whole-body MRI methodology for the objective assessment of inflammation in synovium, tendon, and bone, together with large joint-scoring methodologies for the assessment of inflammation in the hip and knee.

A preliminary scoring method has been developed for whole-body MRI that assesses synovitis and osteitis in 83 peripheral joints and 33 entheses $(0-3$ grading scheme per joint or enthesis) with good reliability attained in a preliminary exercise of 8 patients. The sensitivity to change and discriminatory ability between spondyloarthritis (SpA)

Personal non-commercial use only. The Journal of Rheumatology Copyright $(\subset) 2020$. All rights reserved. 
patients treated with TNF inhibitors and placebo have been demonstrated. This method is expected to be particularly relevant in patients with PsA.

The Hip MRI Inflammation Scoring System (HIMRISS), a granular method for scoring bone marrow lesions in the hip, has been developed and is based on the use of software that applies an overlay to segment the femoral head and acetabulum. This allows multiple regions to be scored directly on a Web-based interface of consecutive MRI slices through the joint (scoring range 0-100), dispensing entirely with the use of scoring spreadsheets. Excellent reliability has been documented in patients with osteoarthritis (OA) of the hip for detection of lesions at a cross-sectional level, and very good reliability has been documented in detecting change 8 weeks after intraarticular glucocorticoid injection in MRI from 90 patients assessed by 8 readers.

The system is now ready for testing in PsA (e.g., assessing the discrimination between treatment groups in clinical trials). Segmentation based on hip joint effusion/synovitis is also performed. A quantitative assessment method, which it is hoped will be superior to semiquantitative assessment, is being developed. Work on automation and comparisons with ultrasound for measuring effusion are also being performed. For the knee, a similar quantitative method, the Knee MRI Inflammation Scoring System (KIMRISS), has been compared with the MRI OA Knee Score system in patients with knee OA and has been shown to have excellent reliability and better reproducibility.

Further, the group has been working on a literature review on the utility of MRI in enthesitis. Lastly, an enthesitis assessment system, including exact definitions of the various pathologies involved, has been developed and validated, with good reproducibility and sensitivity to change. The next steps are to apply the MRI techniques in clinical trials and PsA patient cohorts as well as to further develop and validate the methods on a modular plane.

\section{PRP}

The 2019 annual GRAPPA meeting was the seventh at which PRP have been present. There were 11 PRP who attended (including 2 new members) from North America, South America, Europe, and Asia. The PRP premeeting session included presentations on precision medicine and composite endpoints. PRP were active participants in the meeting's individual and breakout sessions. Individual PRP also presented during the MTX panel debate. The PRP continue to be active in several ongoing GRAPPA activities including the CRN, GRAPPA-OMERACT working group and subgroups, Best Practices, and education.

From a governance perspective, the PRP reported that the PRP Handbook was completed, approved by the GRAPPA Executive Committee, and adopted. Along with the PRP Policies and Procedures, these 2 documents not only define how the PRP group functions and is governed but also support increased group effectiveness. Reflecting the governance, the PRP network has now been able to fully implement the Chair, Chair-elect, and Immediate Past Chair model, with each position held for 2 years.

\section{Education Committee}

As a core objective of GRAPPA's mission, GRAPPA members around the world provide psoriatic disease education to many people, including other healthcare professionals, and patients and their families. On behalf of the Education Committee, Dr. Philip J. Mease discussed a variety of educational activities, including educational symposia, the slide library, and educational videos. He also discussed newer efforts that are aimed at virtual opportunities, such as videoconferencing and podcasts, to reach broader nonrheumatology/dermatology audiences.

In the last decade, many events that have occurred around the world have included accredited continuing medical education (CME) and non-CME symposia. The predominantly US-based CME initiatives have been the GRAPPA-SpA Research and Treatment Network PsA-SpA symposia, offered as 2-h, half-day, and full-day symposia, and the GRAPPA Rheum-Derm PsA-psoriasis symposia. Educational symposia are also conducted around the globe with industry support. Symposia in India have partnered up to 3 international GRAPPA faculty with up to 3 regional faculty and include an ultrasound component. Other events that have occurred or are planned for 2019-2020 include (1) a symposium in Taiwan (November 2019); (2) symposia in Mumbai (November 2019 and February 2020); (3) a symposium in Dubai (September 2019); (4) events at the Asia Pacific League of Associations for Rheumatology meeting [symposium and GRAPPA meeting (April 2019, Brisbane, Australia, and August-September 2020, Kyoto, Japan)]; (5) events at the Pan-American League of Associations for Rheumatology meeting [symposium and GRAPPA meeting (April 2019, Quito, Ecuador, and August 2020, Miami, Florida, USA)]; and (6) events at the African League of Associations for Rheumatology meeting (September 2019, Mauritius).

Guidelines were also provided to qualify an educational symposium as GRAPPA-approved. GRAPPA member involvement is required in planning the symposium agenda. GRAPPA staff could assist with the administrative footwork (e.g., grant applications, identifying audiences, travel logistics, and supporting document creation). GRAPPA faculty tasks include content creation and updates, as well as presentation. Further, participating faculty will have the opportunity to receive mentoring to improve cultural sensitivity during presentations.

GRAPPA aims to provide education in less-resourced areas of the world and is exploring the provision of virtual educational opportunities with videoconferencing and podcasts. Other educational endeavors include the GRAPPA

Personal non-commercial use only. The Journal of Rheumatology Copyright $\subset$ 2020. All rights reserved. 
slide set, which is available for use by members. The popular GRAPPA smartphone application (app) launched in 2018 and is available in 14 languages. Work is ongoing to obtain grant funding to cover the addition of new languages. The Website also continues to host educational content with a plan to provide the GRAPPA videos for members to use for educational and noncertification purposes in the next year.

\section{OMERACT-GRAPPA Outcome Measure Project}

After updating the core domain set for PsA in 2016, the GRAPPA-OMERACT Outcome Measure Working Group has continued its work to develop the core outcome measurement set. The presenters (Dr. Ying Ying Leung, Dr. Ana-Maria Orbai, William Tillett, and Dr. Dafna D. Gladman) reminded attendees that at OMERACT 2018, the $66 / 68$ swollen and tender joint count was endorsed to measure musculoskeletal (MSK) disease activity peripheral arthritis, and the PsA Impact of Disease 12-item questionnaire was provisionally endorsed to evaluate health-related quality of life ${ }^{1,2}$. The working group subsequently prioritized the determination of core measures for 4 additional domains: MSK disease activity enthesitis, physical function, fatigue, and structural damage ${ }^{3}$. Workstreams for each domain are led by a GRAPPA-OMERACT steering committee member and include at least 2 PRP. Identified instruments that assess each domain will be appraised using the OMERACT Filter 2.1 , a set of standards by which to evaluate each instrument for truth, discrimination, and feasibility. Instruments will be proposed for the core outcome measurement set based on the available evidence and will be presented for endorsement by OMERACT. A new endorsement process from OMERACT is planned that will allow approvals through a Web-based portal in real time rather than through the biennial face-to-face OMERACT congress.

\section{Axial Project}

Dr. Gladman and Dr. Denis Poddubnyy presented the ongoing Assessment of SpA international Society (ASAS)-GRAPPA axial project, which aims to develop a consensus definition of axial involvement in PsA. From December 2018 through January 2019, a Web-based survey was conducted among ASAS and GRAPPA members that aimed to identify the variables relevant to the definition of axial involvement in PsA using an established decision-making algorithm [Potentially All Pairwise RanKings of all possible Alternatives (PAPRIKA) method with practical implementation through the 1000 minds Web application]. The survey was completed by 186 ASAS and GRAPPA members (106 ASAS members and 123 GRAPPA members, with 43 members belonging to both societies).

According to the survey results, the following 4 variables that reflect objective signs of inflammatory involvement of the axial skeleton received the highest ranking: (1) presence of structural damage on a radiograph of the sacroiliac joints (radiographic sacroiliitis of at least grade II bilaterally or grade III unilaterally); (2) presence of structural damage on a radiograph of the spine; (3) presence of subchondral bone marrow edema/osteitis on an MRI of the sacroiliac joints compatible with $\mathrm{SpA}$; and (4) presence of bone marrow edema/osteitis on MRI of the spine compatible with SpA. These variables represented the most likely candidates for the future consensus definition. Currently, a prospective study is planned in PsA with comprehensive imaging of the axial skeleton to generate data to validate the expected definition.

\section{Ultrasound Enthesitis Project}

Dr. Lihi Eder presented an update on the GRAPPADiagnostic Ultrasound Enthesitis Tool (DUET) project. The objective of this GRAPPA initiative is to develop a sonographic tool to enable the early diagnosis of PsA, especially because sonography is more sensitive and specific than clinical examination in detecting enthesitis. From pilot studies, a candidate set of entheses was selected. Using these entheses, a larger study is planned within the GRAPPA membership to ensure a diverse population is evaluated to test this potential diagnostic tool. The protocol has been reviewed by the steering committee, and training materials (videos, acquisition atlas) are being developed. A postmeeting workshop was held to understand potential barriers to carrying out studies in various countries. The possibility of performing local reading in addition to the planned central reading was also explored. Members from Europe, Israel, India, as well as North America were present. Challenges within each system were discussed, as well as potential solutions. Timelines to seek study funding were clarified, with the planned study start in mid-2020.

\section{Early Psoriatic Disease Systematic Literature Review}

Dr. Gabriele De Marco reported on the setup of a systematic literature review project entitled "Non-topical pharmacological treatment of early, untreated [disease-modifying antirheumatic drug (DMARD)-naive, systemic therapy-naive] psoriatic disease: a systematic review. " Most trials have been limited with a focus on severe disease of the skin or joints, without looking at more comprehensive indices in outcomes assessment. The target condition was defined so that clinical studies investigating (1) MSK and/or cutaneous manifestations, or (2) untreated psoriatic disease in its early stage could be included. Case reports or studies assessing the effects of topical therapies were excluded. The range of outcome measures considered was broad to assess across the spectrum of disease manifestations and to maximize the sensitivity of the search strategy. The resources accessed to find the available evidence covered a time range from 1946 to May 2019 and spanned electronic databases, trial registers, and conference proceedings. The flow diagram presented during the talk highlighted the substantial number

Personal non-commercial use only. The Journal of Rheumatology Copyright $(\subset) 2020$. All rights reserved. 
of records $(144,299)$ that were identified through electronic database searches. The diagram also provided the status of the ongoing selection activities performed by the team and the timeline for publication.

\section{DISCUSSION}

This paper summarizes GRAPPA's recent work on several projects. These projects are part of GRAPPA's ongoing mission to address educational and unmet research needs for psoriatic disease, to create opportunities for networking within the psoriatic community, and to optimize patient care through collaborative care networks and treatment recommendations.

\section{REFERENCES}

1. Duarte-García A, Leung YY, Coates LC, Beaton D, Christensen R, Craig ET, et al. Endorsement of the $66 / 68$ joint count for the measurement of musculoskeletal disease activity: OMERACT 2018 Psoriatic Arthritis workshop report. J Rheumatol 2019;46: 996-1005.

2. Orbai AM, Holland R, Leung YY, Tillett W, Goel N, Christensen R, et al. PsAID12 provisionally endorsed at OMERACT 2018 as core outcome measure to assess psoriatic arthritis-specific health-related quality of life in clinical trials. J Rheumatol 2019;46:990-5.

3. Leung YY, Orbai AM, Ogdie A, Coates LC, de Wit M, Callis Duffin K, et al. The GRAPPA-OMERACT psoriatic arthritis working group at the 2018 annual meeting: report and plan for completing the core outcome measurement set. J Rheumatol Suppl. 2019 Jun;95:33-7.

4. De Marco G, Berekméri AA, Coates LC, Dubash S, Emmel J, Gladman D, et al. Non topical pharmacological treatment of early, untreated (DMARD-naïve, systemic therapy-näive) psoriatic disease: a systematic review. PROSPERO 2018 CRD42018097366. [Internet. Accessed April 8, 2020.] Available from: www.crd.york. ac.uk/prospero/display_record.php?ID=CRD42018097366. 